\title{
Treatment of smell loss with systemic methylprednisolone*
}

\author{
V.A. Schriever ${ }^{1, \$}$, C. Merkonidis ${ }^{2, \$}$, N. Gupta' ${ }^{1}$, C. Hummel ${ }^{1}$, T. Hummel ${ }^{1}$ \\ Smell \& Taste Clinic, Department of Otorhinolaryngology, University of Dresden Medical School, Dresden, Germany \\ Department of Otolaryngology, Head and Neck Surgery, The Ipswich Hospital NHS Trust, I pswich, United Kingdom
}

\author{
Rhinology 50: 284-289, 2012 \\ DOI:10.4193/Rhino.11.207 \\ *Received for publication: \\ September 20, 2011
}

Accepted: February 5, 2012

\section{Summary}

Background and aim: Olfactory dysfunction is a common complaint in a large number of people. As the aetiologies of olfactory dysfunction vary greatly so do the treatment approaches. The aim of this retrospective study was to evaluate treatment with systemic corticosteroids, particularly focusing on its effectiveness on the different olfactory dysfunction aetiologies. Although a prospective randomized control trail is preferred for such an investigation, using the current approach, we were able to test a very large patient population.

Material and methods: A total of 425 patients with olfactory dysfunction were treated with systemic corticosteroids for 14 days. Olfactory performance was measured using the 'Sniffin' Sticks' battery before and after the treatment.

Results: The treatment with systemic corticosteroids significantly increased the performance on the TDI score and on each of the three subtests; threshold, discrimination and identification. In $26.6 \%$ of the patients improvement of more than six points of the TDI score was observed. The treatment proved to be more effective in patients with sinunasal olfactory dysfunction, where this percentage increased to 36.7 , compared to other aetiologies. In addition, the increase in olfactory function was negatively correlated with the TDI score before the treatment.

Conclusion: This study confirms the effectiveness of systemic corticosteroids on olfactory dysfunction in a large patient population. Specifically, the results show that treatment is: (a) more effective in patients with sinunasal than in patients with idiopathic olfactory dysfunction, (b) most effective in patients with sinunasal disease with nasal polyps, and (c), at best, effective in half of the patients. The current study may provide help in counselling patients.

Key words: sinunasal disease, inflammation, smell, nose, methylprednisolone

\section{Introduction}

Many people are affected by olfactory dysfunction. It is estimated that in Germany every year approximately 50,000 patients with olfactory dysfunction seek specialist help ${ }^{(1)}$. It is presumed that the actual number of people with olfactory dysfunction is even higher because patients are often unaware of their condition. For example, according to Nordin et al., up to $77 \%$ of elderly people with smell loss do not report olfactory problems ${ }^{(2)}$.

Olfactory dysfunction is caused by a variety of aetiologies. The most common reasons for olfactory loss found in smell and taste clinics are: sinunasal, post infectious, idiopathic, post traumatic, iatrogenic, toxic, and congenital ${ }^{(1)}$. In addition, it is known that many other reasons such as neurodegenerative diseases or age can cause olfactory dysfunction ${ }^{(3,4)}$. A study by Damm et al. showed that sinunasal olfactory dysfunction with $72 \%$ is by far the most frequent aetiology being followed by post infectious $(11 \%)$ and idiopathic (6\%) causes. These numbers vary in relation to the source of the survey, for example in some specialised smell and taste clinics the number of post infectious olfactory dysfunction reaches $91 \%$ of all patients ${ }^{(1)}$. 
Although the number of patients suffering of olfactory dysfunction is large, there is still no universally accepted treatment protocol available. As a result, there are many different treatment suggestions for olfactory dysfunction. These can be summarised in three major groups: surgical, pharmacological, and others. The surgical approach addresses mainly sinunasal olfactory dysfunctions. The outcome of this treatment is rather modest. Studies have shown, that after surgery the sense of smell improved only in $25 \%$ of the pre surgical hyposmic and $5 \%$ of the pre surgical anosmic patients ${ }^{(5,6)}$. A recent review on this topic by Rudmik and Smith reported olfactory improvement after endoscopic sinus surgery in $0-75 \%$ of the patients ${ }^{(7)}$. In this study, predictive factors for the outcome of endoscopic sinus surgery on the olfactory function, such as eosinophilia or preoperative anosmia, were also identified ${ }^{(7)}$.

Other approaches to treat olfactory dysfunction include olfactory training. Again, with this method approximately $25 \%$ of the patients show an improvement of olfactory function ${ }^{(8)}$.

The most commonly used treatment modality in olfactory dysfunction is pharmacological. From the wide range of substances that are in use, only a few have been found to be effective. For example, the effectiveness of vitamins and of antiviral agents is controversial ${ }^{(1,9,10)}$.

Corticosteroids are the most frequently used drugs in the treatment of olfactory loss ${ }^{(11)}$. There are two ways of corticosteroid application; topically in the nasal cavity and systemic. With the former it is hoped to prevent the systemic side effects of corticosteroids. An improvement of the sense of smell has been found for both topical (12-14) and systemic application ${ }^{(1,15,16)}$ of corticosteroids.

In this study, we examined the effects of systemic corticosteroids on the sense of smell in a population of 425 patients. The study focuses on the comparison of the effectiveness of the treatment in the various aetiologies of olfactory dysfunction and in particular the sinunasal and idiopathic aetiologies. It is hoped that this retrospective study will contribute to better understanding and applying of this treatment modality.

\section{Materials and methods}

The retrospective study was approved by the institutional review boards of the University of Dresden Medical School, Germany (EK number 251112006), and the The Ipswich Hospital, UK, respectively. The study was designed to examine effects of the treatment of olfactory dysfunction with systemic corticosteroids.

\section{Patients}

A total of 425 patients with subjective olfactory dysfunction (213 females and 212 males) with an age range from 14 to 85 years (mean \pm standard deviation $(S D)=56.6 \pm 12.1$ years) from the Smell and Taste Clinic in Dresden and The Ipswich Department of ORL were included in this study. The data was collected between $07 / 2000$ and 12/2010.

\section{Inclusion criteria}

To evaluate the effectiveness of systemic corticosteroid treatment on the olfactory function, patients with olfactory loss due to sinunasal, idiopathic, post-traumatic, post-infectious, and post-surgical aetiologies were included in the study. Because patients were unequally distributed among the different aetiologies, it was only possible to compare treatment between three aetiology groups: sinunasal, idiopathic and post-infectious. In addition, all patients included in this study suffered from subjective olfactory dysfunction.

\section{Procedure}

Every patient had at least two visits to the clinic; before and after systemic treatment with corticosteroids. A detailed medical history was obtained at the patients' first visit with a focus on olfactory dysfunction followed by a clinical examination of the upper respiratory tract including an endoscopy of the nose. Hence, the aetiology of the olfactory dysfunction was determined for each patient. The aetiologies were classified in three groups: sinunasal $(n=221)$, idiopathic $(n=157)$ and other causes $(n=47)$, e.g., post traumatic, post infectious etc. (Figure 1). If no reason for the olfactory dysfunction could be found, the patient was included in the idiopathic group. Thus, this group is very heterogeneous and might include a variety of undetected causes of olfactory dysfunction. The mean duration of the symptoms was $67 \pm 76$ months at the time of the first visit. The majority - 253 patients - had olfactory dysfunction for more than 24 months; 172 patients had the symptoms for less than two years. On each visit, the patients' olfactory function was assessed. The second visit was scheduled at the last day of treatment.

\section{Olfactory testing}

The 'Sniffin' Sticks' were used to evaluate olfactory function. This olfactory test battery consists of three tests: (a) phenyl ethyl alcohol odour thresholds ( $T$ ), (b) odour discrimination (D), and (c) odour identification (I). The maximum score for each test was 16 points resulting to a maximum sum of 48 points (TDI score) for the three tests. The 'Sniffin' Sticks' test allows for a classification of olfactory function as normosmia, hyposmia, or functional anosmia ${ }^{(17,18)}$.

\section{Treatment}

Patients received systemic treatment with methylprednisolone for 14 days. The starting daily dose was $40 \mathrm{mg}$ p.o. and the dosage was reduced by $5 \mathrm{mg}$ every other day. Above corticosteroid and its dosing regimen has been part of our management protocol of patients with suspected olfactory dysfunction due to inflammatory causes for more than 20 years with no major 


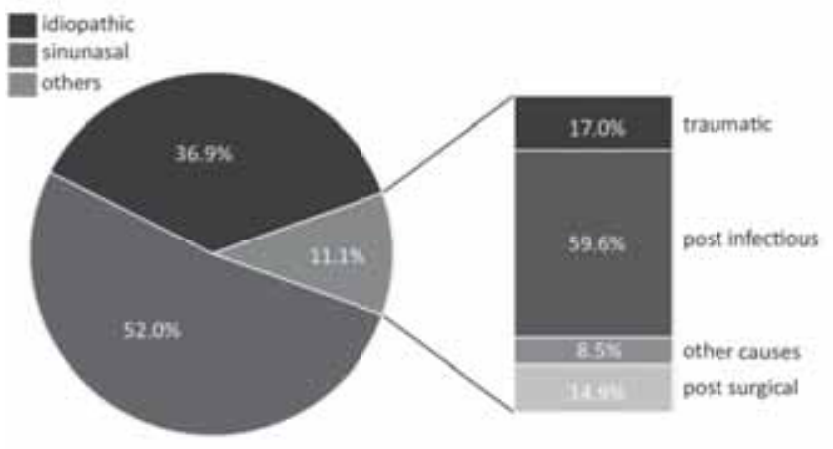

Figure 1. Aetiology of the patients' olfactory dysfunction. The diagram shows the aetiology of the olfactory function for the 425 patients treated in this study. $52.0 \%$ of all patients had sinunasal olfactory dysfunction. For $36.9 \%$ the aetiology was idiopathic and $11.1 \%$ had other aetiologies. The reasons of the olfactory dysfunction of the $11.1 \%$ are shown in the right part of the diagram.

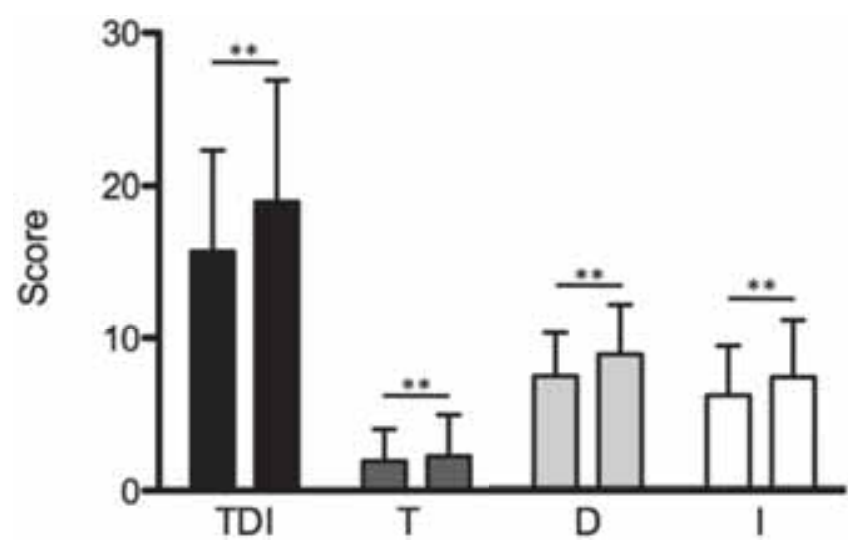

Figure 2. Effects of methylprednisolone on the TDI score. The Figure shows the TDI scores before and after treatment with methylprednisolone. The left bars show the change in TDI score. Subtest T (threshold), D (discrimination) and I (identification) are represented by the bars to the right. The treatment caused a significant change in the TDI score as well as in the subtests $\left(^{* *}=p<0.001\right)$; interestingly, all subtests responded similarly to treatment.

problems reported so far. Our choice of corticosteroid, its total dose and dosing regimen were based on our positive experiences with this treatment modality. Moreover, to our knowledge, there are no comparative studies on the effectiveness of the different corticosteroid doses in restoring olfactory function. Detailed information about the treatment and the possible side effects of methylprednisolone was given to the patients before onset of treatment.

\section{Statistical analysis}

The data was analysed using SPSS 19 (SPSS Inc., Chicago, IL, USA). Paired t-test and correlations using Pearson statistics were used to examine the influence of the treatment with methylprednisolone on the TDI scores. The a level was set to 0.05 .

\section{Results}

A total of 425 patients were included in this study. The majority of these patients were functionally anosmic $(55.1 \% ; n=234)$ or hyposmic ( $40.9 \% ; n=174)$; only $4 \%(n=17)$ were normosmic. Although these 17 patients were normosmic according to the TDI score, they complained about subjective olfactory dysfunction. None of the patients reported major side effects caused by the treatment. The average TDI score for all patients before treatment was 15.7 points ( $S D=6.55)$. After treatment with methylprednisolone the TDI score improved significantly by on average 3.25 points $(S D=6.46)$ to 18.95 points $(S D=7.94)(t=$ $10.4, \mathrm{p}<0.001$ ) (Figure 2).

All three sub-scores of the 'Sniffin' Sticks' battery showed a significant increase after treatment. Scores in the threshold test increased from $1.93(S D=2.03)$ to $2.27(S D=2.63)(t=5.94, p<$ $0.001)$. In the odour discrimination test the increase ranged from $7.52(S D=2.83)$ to 8.95 points $(S D=3.23)(t=9.00, p<0.001)$. The scores in the odour identification test increased from 6.24 $(\mathrm{SD}=3.27)$ to $7.43(\mathrm{SD}=3.76)(\mathrm{t}=7.22, \mathrm{p}<0.001)$ (Figure 2$)$.

Previous studies have reported the importance of a six or more point increase of the TDI score for the subjective improvement of the olfactory function ${ }^{(19)}$. As shown in Figure $3 a$, in our study $26.6 \%(n=113)$ of patients showed a TDI score increase of six points or more. In contrast, only $3.5 \%(n=15)$ of patients showed a deterioration of the TDI score by six or more points after treatment. In 69.9\% ( $n=297)$, the treatment resulted in an increase of the TDI score of no more than six points.

The TDI score before treatment was negatively correlated with the increase of the TDI score after treatment $(r=0.25, p=0.01)$, indicating that improvement was higher in subjects with a low pre-treatment TDI score. In other words, patients with functional anosmia ( $\mathrm{t}=9.90, \mathrm{p}<0.001)$ and hyposmia $(\mathrm{t}=4.39, \mathrm{p}<0.001)$ improved to a higher degree than normosmic patients where, in fact, no significant effect of treatment was seen $(t=0.26$, $p=0.80$ ). Neither gender, age nor the length of the olfactory dysfunction were found to have any influence on the outcome of the systemic treatment with methylprednisolone.

We found that the aetiology of the olfactory dysfunction had an impact on the outcome of the treatment. As described above, we divided the patients according to their history into three aetiological groups: sinunasal $(n=221)$, idiopathic $(n=157)$, and other causes $(n=47)$. As the causes of olfactory loss in the third group were very heterogeneous, the focus of this analysis was on the post infectious group $(n=27)$, which had been extracted from the 'other causes' group. In all three groups treatment increased 


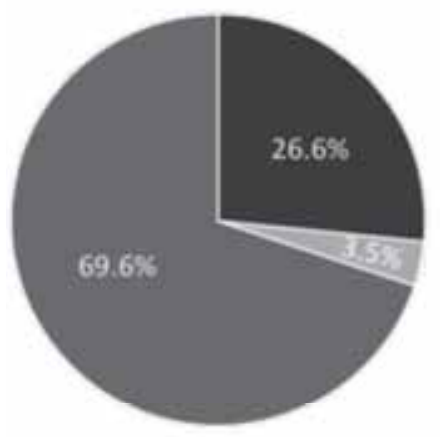

a) all etiologies

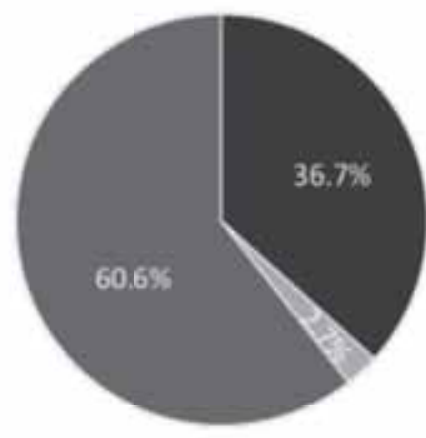

b) sinunasal

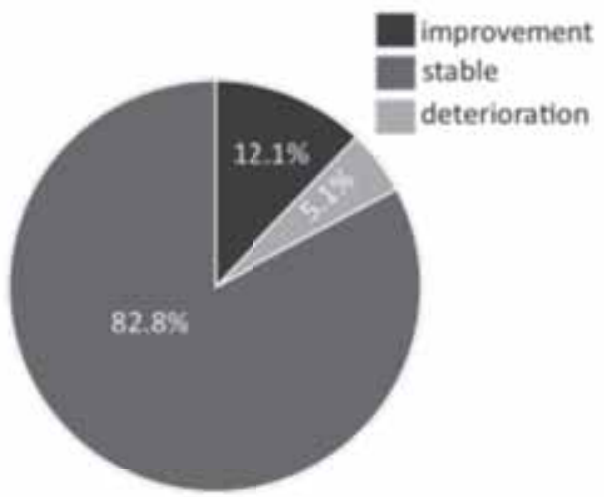

c) idiopathic

Figure 3. Effectiveness of the treatment considering the aetiology of the olfactory dysfunction. The figure shows the effectiveness of the treatment with methylprednisolone. An improvement is considered as an increase of the TDI score of six or more points. Deterioration means a decrease of the DTI score of 6 points or more and stable stands for a change less than six points. a) all patients, b) patients with sinunasal olfactory dysfunction, c) patients with idiopathic olfactory dysfunction. For $36.7 \%$ of the patients with sinunasal olfactory dysfunction the TDI score improved six points of more. Only $12.1 \%$ of patients with idiopathic olfactory dysfunction showed this improvement.

the TDI scores significantly. The differences in results between these aetiologies will be pointed out in the discussion below.

For the patients in the idiopathic group, the TDI score increased with an average of 1.0 point ( $S D=4.78)$ from 14.71 ( $S D=5.47$ ) to $15.71(S D=5.99)(t=2.62, p=0.01)$. No significant increase of the olfactory threshold was observed $(t=0.13, p=0.90)$. Only $12.1 \%$ of this group showed an improvement - an increase of sixc or more points on the TDI score (Figure 3c). The TDI score remained stable in $82.8 \%$ of patients. In $5.1 \%$, the score deteriorated by six or more points. As pointed out in the'all patients group,' gender, age or the length of the olfactory dysfunction had no influence on the results of the treatment.

In the group of post-infectious olfactory dysfunction, the TDI scores improved by on average 4.47 points $(S D=7.09)$ i.e from $14.39(\mathrm{SD}=6.61)$ to $18.86(\mathrm{SD}=8.41)(\mathrm{t}=3.28, \mathrm{p}=0.003)$. All three sub-scores of the TDI showed a significant increase. Since the number of patients in this group was relatively small ( $\mathrm{n}=$ 27) it was not possible to determine the influence of gender, age or length of olfactory dysfunction on the outcome. $29.6 \%$ of the patients in this group showed an increase of six or more points in the TDI score. The score stayed stable for $70.4 \%$ and no deterioration of the TDI score was found in any of the patients in this group. Further studies are needed to address this group of patients separately in order to optimize treatment.

Patients with sinunasal olfactory dysfunction showed an average increase of the TDI score of 4.79 points $(S D=6.92)$ i.e. from $16.65(S D=7.07)$ to $21.45(S D=8.10)(t=10.29, p<0.001)$. Scores in the three subtests, threshold $(t=6.15, p<0.001)$, discrimination $(t=9.40, p<0.001)$ and identification $(t=6.62$, $\mathrm{p}<0.001$ ) all increased significantly. In line with the other aetiology groups, no influence of gender, age or the length of olfactory dysfunction was observed in this group. Individual improvement was found in $36.7 \%$ of this group all with an increase of their TDI score by six or more points (Figure $3 \mathrm{~b}$ ). In $60.6 \%$, the TDI score remained stable and in $2.7 \%$ the score deteriorated by six or more points. Before treatment, $49.3 \%$ of the patients with sinunasal olfactory dysfunction were anosmic. After treatment, $50.5 \%$ of them were found to be hyposmic and $6.4 \%$ improved to normal olfactory function. Patients who were hyposmic before the treatment in this group had a higher rate of regaining normal olfactory function (21.0\%).

Sinunasal olfactory dysfunction can be divided further into at least two subgroups, patients with and patients without nasal polyps. Patients with nasal polyps $(n=68)$ showed a large increase in the TDI score of on average 9.06 points $(S D=9.20)$ i.e. from $16.36(S D=9.22)$ to 25.43 points $(S D=10.15)(t=4.47$, $\mathrm{p}<0.001)$. It is interesting that no significant increase of the olfactory threshold was observed $(t=1.56, p=0.13)$. Scores in the discrimination $(t=5.85, p<0.001)$ and the identification test $(\mathrm{t}=3.31, \mathrm{p}=0.003$ ) both increased significantly with treatment. $50.0 \%$ of this group showed an increase of the TDI score of six or more points. For $5.0 \%$ the TDI score deteriorated by six or more points and the rest (45.0\%) showed stable TDI scores. In addition to the large increase of TDI score, $18.2 \%$ of the pre-treatment anosmic patients and $57.1 \%$ of the pre-treatment hyposmic patients were considered normosmic after treatment. Unfortunately, in this retrospective study no data were available that 
would have allowed for a more refined definition of subgroups of patients with polyps.

Although the treatment increased significantly, the TDI score in all aetiology groups, it was more effective in patients with sinunasal olfactory dysfunction. The average TDI increase in patients with sinunasal olfactory dysfunction (4.79 (SD = 5.45)) was significantly higher when compared to all other patients (1.57 $(S D=6.92))(t=5.35, p<0.001)$. Not only was the average increase in this group higher, but also more patients with sinunasal olfactory dysfunction showed an increase of six or more points on the TDI score (36.7\% compared to $15.7 \% ; x^{2}=23.9, p<0.001$ ). In addition, more patients with sinunasal olfactory dysfunction regained a normal sense of smell (13.4\%) compared to patients suffering from other aetiologies of olfactory dysfunction (3.5\%) $\left(x^{2}=13.6, p<0.001\right)$

\section{Discussion}

With this retrospective study, we substantiated the effectiveness of systemic corticosteroids in the treatment of olfactory dysfunction in a very large group of patients $(n=425)$. We found, that the TDI score increased significantly after treatment. In addition, all individual sub-scores of the TDI increased significantly. However, on an individual level improvement was only found in an approximately one third of the patients when looking at the different aetiologies. This number was greatly increased when nasal polyps were taken as a factor. In this group 50\% showed an improvement of six or more point in the TDI score.

Further to any previous studies, we applied high threshold criteria to the effectiveness of the treatment. Only an increase of the TDI score by six or more points was considered as an improvement of olfactory function ${ }^{(19)}$. This criterion was fulfilled in $26.6 \%$ of our patients.

In our further analysis, we focused on sinunasal and idiopathic olfactory dysfunction, due to the inhomogeneous distribution of aetiologies in the patient population. We were able to show that the treatment with methylprednisolone is more effective in patients with sinunasal olfactory dysfunction than in patients with idiopathic olfactory dysfunction. We observed a significantly higher increase of the TDI score in the sinunasal group compared to the idiopathic group with a higher proportion of individual patients from the sinunasal group exhibiting improvement. More specifically, improvement was found in $36.7 \%$ of patients with sinunasal disease compared to $12.1 \%$ of patients with idiopathic olfactory loss. Therefore, treatment with systemic corticosteroids should not be considered the first option in the management of patients with idiopathic olfactory dysfunction. We also observed that the patients with a low pre-treatment TDI score showed a greater increase in olfactory function.

Even though idiopathic olfactory dysfunction was diagnosed very carefully, it might be that patients with undetected sinunasal or other causes of olfactory dysfunction were included in this group. These patients are expected to respond better to treatment with systemic corticosteroids compared to patients with real idiopathic olfactory dysfunction.

\section{Corticosteroids are widely used as anti-inflammatory medica-} tion. Their exact mechanism of action in patients with olfactory dysfunction is not yet known. There is some evidence that glucocorticoid receptors and the adenosine triphosphatase may play a role ${ }^{(20-22)}$. Furthermore, one proteomics study indicated that the administration of glucocorticoids triggers the apoptosis pathway in nasal polyps ${ }^{(23)}$. Patients with nasal polyps benefit a great deal from treatment with corticosteroids in terms of airflow, although, not all of these patients benefit in terms of olfactory function. Why some patients with idiopathic olfactory loss responded to corticosteroid treatment is still subject to speculation. It is likely that a small portion of this group exhibits inflammation, which is not seen macroscopically.

Comparison between surgical treatment and pharmacological treatment is difficult. The numbers for improvement of olfactory function with surgical treatment vary greatly between studies. Bonfils et al., reported improvement of olfactory function in $13-91 \%$ with a mean of $31 \%$ of the patients responding ${ }^{(24)}$. Part of this deviation between studies is due to the fact that in most, evaluation of the sense of smell was based only on questioning the patients without performing smell tests. Results of these studies are difficult to interpret as most people typically confuse changes in respiratory function with changes in olfactory function ${ }^{(25)}$. When measuring olfactory function, Delank et al., found an improvement in $25 \%$ of their patients, which is in line with the mean of $31 \%$ in Bonfils' review ${ }^{(5)}$. Finally, an excellent study by Blomqvist et al., shows no benefit of a surgical treatment over treatment with corticosteroids ${ }^{(26)}$. These divergent data suggest a careful application of the surgical treatment. Thus, it appears that overall, surgical and conservative treatment produce similar rates of olfactory improvement.

The treatment protocol used in this study starting methylprednisolone at $40 \mathrm{mg}$ and reducing the dosage by $5 \mathrm{mg}$ every other day has shown to be effective in the treatment of olfactory dysfunction, especially in cases with a sinunasal aetiology. However, more research is required to optimize the treatment protocol on the aetiology of the olfactory dysfunction.

With the retrospective design of this study, we were able to show the effectiveness of corticosteroids in the treatment ofolfactory dysfunction in a very large patient population. A prospective randomized control trail should be considered for further investigations to examine the benefit of this treatment. 


\section{Conclusion}

This study confirms the effectiveness of systemic corticosteroids on olfactory dysfunction in a large patient population. Specifically, the results show that treatment is (a) more effective in patients with sinunasal than in patients with idiopathic olfactory dysfunction, (b) most effective in patients with sinunasal disease with nasal polyposis, and (c), at best, effective in half of the patients. We hope that the results of the current study will provide help in counselling patients.

\section{Acknowledgements}

This study was supported by a grant from the FNRS Fonds de la Recherche Scientifique, Brussels, to TH.

\section{Authorship contribution}

Data collection and patient treatment: MC, TH. Data analysis: VAS, $\mathrm{CH}$, TH. Writing the manuscript: VAS, $\mathrm{CM}, \mathrm{NG}, \mathrm{CH}, \mathrm{TH}$.

\section{Conflict of interest}

None of the authors declares a conflict of interest.

\section{References}

1. Damm M, Temmel A, Welge-Lussen A, et al. Olfactory dysfunctions. Epidemiology and therapy in Germany, Austria and Switzerland. HNO. 2004; 52: 112-120.

2. Nordin S, Monsch AU, Murphy $C$. Unawareness of smell loss in normal aging and Alzheimer's disease: discrepancy between self-reported and diagnosed smell sensitivity. J Gerontol B Psychol Sci Soc Sci. 1995; 50: P187-192.

3. Nordin S, Bramerson A. Complaints of olfactory disorders: epidemiology, assessment and clinical implications. Curr Opin Allergy Clin Immunol. 2008; 8: 10-15.

4. Holbrook EH, Leopold DA. An updated review of clinical olfaction. Curr Opin Otolaryngol Head Neck Surg. 2006; 14: 23-28.

5. Delank KW, Stoll W. Olfactory function after functional endoscopic sinus surgery for chronic sinusitis. Rhinology. 1998; 36: 15-19.

6. Pade J, Hummel T. Olfactory function following nasal surgery. Laryngoscope. 2008; 118: 1260-1264.

7. Rudmik L, Smith TL. Olfactory improvement after endoscopic sinus surgery. Curr Opin Otolaryngol Head Neck Surg. 2011. Dec. [Epub ahead of print].

8. Hummel T, Rissom K, Reden J, Hahner A, Weidenbecher M, Huttenbrink KB. Effects of olfactory training in patients with olfactory loss. Laryngoscope. 2009; 119: 496-499.

9. Garrett-Laster M, Russell RM, Jacques PF. Impairment of taste and olfaction in patients with cirrhosis: the role of vitamin A. Hum Nutr Clin Nutr. 1984; 38: 203-214.

10. Heilmann S, Just T, Goktas O, Hauswald B, Huttenbrink KB, Hummel T. Effects of systemic or topical administration of corticosteroids and vitamin B in patients with olfactory loss. Laryngorhinootologie. 2004; 83: 729-734.

11. Hummel T, Landis BN, Huttenbrink KB. Dysfunction of the chemical senses smell and taste. Laryngorhinootologie. 2011; 90 Suppl 1: S44-55.

12. Meltzer EO, Jalowayski AA, Orgel HA, Harris AG. Subjective and objective assessments in patients with seasonal allergic rhinitis: effects of therapy with mometasone furoate nasal spray. J Allergy Clin Immunol. 1998; 102: 39-49.

13. Stuck BA, Blum A, Hagner AE, Hummel $T$, Klimek L, Hormann K. Mometasone furoate nasal spray improves olfactory performance in seasonal allergic rhinitis. Allergy. 2003; 58: 1195.

14. Mott AE, Cain WS, Lafreniere D, Leonard G, Gent JF, Frank ME. Topical corticosteroid treatment of anosmia associated with nasal and sinus disease. Arch Otolaryngol Head Neck Surg. 1997; 123: 367-372.

15. Hotchkiss WT. Influence of prednisone on nasal polyposis with anosmia; preliminary report. AMA Arch Otolaryngol. 1956; 64: 478-479.

16. Fein BT, Kamin PB, Fein NN. The loss of sense of smell in nasal allergy. Ann Allergy. 1966; 24: $278-283$.

17. Hummel T, Sekinger B, Wolf SR, Pauli E, Kobal G. 'Sniffin' sticks': olfactory performance assessed by the combined testing of odor identification, odor discrimination and olfactory threshold. Chem Senses. 1997; 22: 39-52.

18. Hummel T, Kobal G, Gudziol H, Mackay-Sim A. Normative data for the "Sniffin' Sticks" including tests of odor identification, odor discrimination, and olfactory thresholds: an upgrade based on a group of more than 3,000 subjects. Eur Arch Otorhinolaryngol. 2007; 264: 237-243.

19. Gudziol V, Lotsch J, Hahner A, Zahnert T, Hummel T. Clinical significance of results from olfactory testing. Laryngoscope. 2006; 116: 1858-1863.

20. Robinson AM, Kern RC, Foster JD, Fong KJ, Pitovski DZ. Expression of glucocorticoid receptor $\mathrm{mRNA}$ and protein in the olfactory mucosa: physiologic and pathophysiologic implications. Laryngoscope. 1998; 108 (8 Pt 1): 1238-1242.

21. Robinson AM, Kern RC, Foster JD, Krozowski ZS, Pitovski DZ. Mineralocorticoid receptors in the mammalian olfactory mucosa. Ann Otol Rhinol Laryngol. 1999; 108: 974-981.

22. Fong KJ, Kern RC, Foster JD, Zhao JC,
Pitovski DZ. Olfactory secretion and sodium, potassium-adenosine triphosphatase: regulation by corticosteroids. Laryngoscope. 1999; 109: 383-388.

23. Farajzadeh Deroee A, Oweinah J, Naraghi M, et al. Regression of polypoid nasal mucosa after systemic corticosteroid therapy: a proteomics study. Am J Rhinol Allergy. 2009; 23: 480-485.

24. Bonfils $P$, Malinvaud D, Soudry Y, Devars du Maine M, Laccourreye O. Surgical therapy and olfactory function. B-ENT. 2009; 5 Suppl 13: 77-87.

25. Landis BN, Hummel T, Hugentobler M, Giger R, Lacroix JS. Ratings of overall olfactory function. Chem Senses. 2003; 28: 691-694.

26. Blomqvist EH, Lundblad L, Anggard A, Haraldsson PO, Stjarne P. A randomized controlled study evaluating medical treatment versus surgical treatment in addition to medical treatment of nasal polyposis. J Allergy Clin Immunol. 2001; 107: 224-228.

27. Hummel T, Stuck BA. Treatment of olfactory disorders. HNO. 2010; 58: 656-660.

28. Reden J, Herting B, Lill K, Kern R, Hummel T. Treatment of postinfectious olfactory disorders with minocycline: a double-blind, placebo-controlled study. Laryngoscope 2011; 121: 679-682.

Thomas Hummel, M.D.

Smell \& Taste Clinic

Department of Otorhinolaryngology

University of Dresden Medical School

Fetscherstr. 74

01307 Dresden

Germany

Tel: +49-351-458-4189

Fax: +49-351-458-7370

E-mail: thummel@mail.zih.tu-dresden.de 